\title{
Entropic gravity versus gravitational pseudotensors in static spherically symmetric spacetimes
}

\author{
S. Hamid Mehdipour ${ }^{1, *}$ \\ ${ }^{1}$ Department of Physics, College of Basic Sciences, Lahijan Branch, \\ Islamic Azad University, P. O. Box 1616, Lahijan, Iran
}

(Dated: October 26, 2018)

\begin{abstract}
We present some well-known energy-momentum complexes and evaluate the gravitational energy associated with static spherically symmetric spacetimes. In fact, the energy distribution of the aforementioned gravitational background that is contained in a two-sphere of radius $r$ shows that a test particle situated at a finite distance $r$ experiences the gravitational field of the effective gravitational mass. In addition, we apply Verlinde's entropic gravity to find the emergent gravitational energy on static spherically symmetric screens. In this setup, we find that the energy distribution in the prescription of Møller is similar to the energy derived from the emergent gravity, while other prescriptions give the different results. This result may confirm the argument of Lessner who argues that Møller's definition of energy is a powerful concept of energy in General Relativity.

PACS numbers: 04.20.-q, 05.70.-a, 04.70.Dy

Keywords: Energy Distribution, Pseudotensor, Entropic Gravity, Holographic Screen
\end{abstract}

*Electronic address: mehdipour@liau.ac.ir 


\section{INTRODUCTION}

The notion of energy has been an issue of extensive research since the beginning of Einstein's theory of General Relativity (GR). Einstein [1] was the first to consider a locally conserved formula for energy-momentum complexes including the contribution from the gravitational field energy. He obtained an expression for the energy-momentum complex by introducing the gravitational field pseudotensor. This idea followed by a surge of interest and the various prescriptions for the energy-momentum complexes as tools to determine energy-momentum distributions were suggested [2 7]. These prescriptions were confined to evaluate the energy and momentum in quasi-Cartesian coordinates. Afterwards, a new description for the energy-momentum complex was proposed by Møller [8] which is not restricted to quasi-Cartesian coordinates and furthermore it provides a powerful concept of energy and momentum in GR [9]. Nevertheless, the topic of energy-momentum complexes has been fundamentally disputed in the context of GR (see [10] and the references therein). There are many unanswered questions regarding the energy and momentum localization in the literature (see for instance [11]). There have been a lot of attempts to obtain a wellbehaved expression for local or quasi-local energy-momentum and a number of studies have been performed on this debatable issue [12].

In this paper, we will evaluate the gravitational energy associated with static spherically symmetric (SSS) spacetimes. We employ two approaches. The first one is the pseudotensor approach and the other one is the entropic approach. It is evident that there exists a link between gravity and thermodynamics. Jacobson [13] has exhibited that the Einstein field equations of GR are derived from the first law of thermodynamics. Padmanabhan [14] has applied the equipartition law of energy and the holographic principle to present a thermodynamic explanation of gravity (see also [15]). A recent work by Verlinde [16] has suggested a novel approach to interpret the gravity as an entropic force owing to alterations in the information connected to the positions of material bodies. In this approach, one could imagine an emergent phenomenon for the origin of Newtonian gravity. This theory indicates that the gravitational interaction arises from the statistical behavior of microscopic degrees of freedom encoded on a holographic screen. The entropic approach has recently given an impetus for more study in the literature [17].

The paper is organized as follows. In Sec. II, using the energy-momentum definitions 
of Einstein, Landau-Lifshitz, Weinberg, Papapetrou and Møller, we calculate the energy distribution of the SSS spacetimes in a generic form, respectively. In Sec. III, we study Verlinde's idea about the temperature and the energy on the holographic screens for generic spherically symmetric surfaces. Finally, we present a summary in Sec. IV] In this work, we will choose to write spacetime indices using the Greek alphabet, and space indices using the Latin alphabet. We will also choose to use natural units, where $\hbar=c=G=k_{B}=1$.

\section{ENERGY-MOMENTUM COMPLEXES}

The energy-momentum conservation in the context of GR can be written as

$$
\nabla_{\mu} T_{\nu}^{\mu}=0,
$$

where $T_{\nu}^{\mu}$ is the symmetric energy-momentum tensor containing the matter and all nongravitational fields. Einstein [1] conjectured a energy-momentum complex $\mathcal{T}_{\nu}^{\mu}$ containing the matter, all non-gravitational fields and the gravitational field such that it obeys a conservation law in the form of a divergence in the following form

$$
\mathcal{T}_{\nu, \mu}^{\mu}=0,
$$

with

$$
\mathcal{T}_{\nu}^{\mu}=\sqrt{-g}\left(T_{\nu}^{\mu}+t_{\nu}^{\mu}\right),
$$

where comma indicates partial differentiation and $g$ is the determinant of the metric tensor $g_{\mu \nu}$. The expression $t_{\nu}^{\mu}$ exhibits the energy-momentum pseudotensor which is a nontensorial quantity to describe the gravitational field energy. We can also write the energy-momentum complex in the following form

$$
\mathcal{T}_{\nu}^{\mu}=\theta_{\nu, \lambda}^{\mu \lambda},
$$

where $\theta_{\nu}^{\mu \lambda}$ is denoted as superpotential components which are functions of the metric tensor and its first order derivatives. It is obvious that the energy-momentum complex does not have a unique definition due to the fact that one may always add a quantity with a zero divergence to the expression $\mathcal{T}_{\nu}^{\mu}$.

We are interested in computing the energy distribution associated with the SSS gravitational background, which is contained in a two-sphere of radius $r$. Thus, the background 
metric is supposed to be a generic SSS solution as follows:

$$
d s^{2}=-A(r) d t^{2}+B(r) d r^{2}+r^{2} d \Omega^{2}
$$

where $A$ and $B$ are arbitrary functions of the radial coordinate and $d \Omega^{2}=d \theta^{2}+\sin ^{2} \theta d \varphi^{2}$ gives the standard line element on the unit two-sphere. For carrying out the calculations with Einstein, Landau-Lifshitz, Weinberg and Papapetrou energy-momentum complexes, we require to reexpress the SSS metric in quasi-Cartesian coordinates. Transforming (15) to Cartesian terms according to $x=r \sin \theta \cos \varphi, y=r \sin \theta \sin \varphi$, and $z=r \cos \theta$, one gets the metric

$$
d s^{2}=-A(r) d t^{2}+d x^{2}+d y^{2}+d z^{2}+\frac{B(r)-1}{r^{2}}(x d x+y d y+z d z)^{2},
$$

where $r^{2}=x^{2}+y^{2}+z^{2}$. In the following subsections, we will present some well-known energy-momentum complexes for obtaining the gravitational energy in SSS spacetimes.

\section{A. Energy distribution in Einstein's prescription}

Einstein's energy-momentum complex [1] has the form

$$
\theta_{\nu}^{\mu}=\frac{1}{16 \pi} h_{\nu, \lambda}^{\mu \lambda}
$$

where Einstein's superpotential $h_{\nu}^{\mu \lambda}$ is given by

$$
h_{\nu}^{\mu \lambda}=\frac{1}{\sqrt{-g}} g_{\nu \sigma}\left[-g\left(g^{\mu \sigma} g^{\lambda \kappa}-g^{\lambda \sigma} g^{\mu \kappa}\right)\right]_{, \kappa},
$$

with the antisymmetric property

$$
h_{\nu}^{\mu \lambda}=-h_{\nu}^{\lambda \mu}
$$

The energy in Einstein's prescription for a four-dimensional background is given by

$$
E=\iiint \theta_{0}^{0} d x^{1} d x^{2} d x^{3}
$$

where $\theta_{0}^{0}$ is the energy density of the total physical system including gravitation. The integrals in Eq. (10) are extended over all space for $x^{0}=$ const. Using Gauss's theorem, the energy component is equal to

$$
E=\frac{1}{16 \pi} \iint h_{0}^{0 i} n_{i} d S
$$


where $n_{i}=x_{i} / r$ is the outward unit normal vector over an infinitesimal surface element $d S$. Using Eq. (11) and evaluating the integrals over the surface of two-sphere of radius $r$, the energy distribution associated with the generic SSS metric in Einstein's formulation is found to be

$$
E_{E}=\frac{r A(B-1)}{2 \sqrt{A B}}
$$

\section{B. Energy distribution in Landau-Lifshitz's prescription}

The energy and momentum in the prescription of Landau-Lifshitz [4] is given by

$$
L^{\mu \nu}=\frac{1}{16 \pi} \mathcal{S}_{, \lambda \kappa}^{\mu \nu \lambda \kappa}
$$

with

$$
\mathcal{S}^{\mu \nu \lambda \kappa}=-g\left(g^{\mu \nu} g^{\lambda \kappa}-g^{\mu \lambda} g^{\nu \kappa}\right)
$$

where $L^{\mu \nu}$ is symmetric with respect to its indices. Landau-Lifshitz's superpotential $\mathcal{S}^{\mu \nu \lambda \kappa}$ has symmetries similar to the curvature tensor. The energy in the Landau-Lifshitz prescription for a four-dimensional background is given by

$$
E=\iiint L^{00} d x^{1} d x^{2} d x^{3},
$$

where $L^{00}$ is the energy density component. Using Gauss's theorem, the energy component is

$$
E=\frac{1}{16 \pi} \iint \mathcal{S}_{, \kappa}^{00 i \kappa} n_{i} d S .
$$

Using the metric (6), we get the energy distribution in Landau-Lifshitz's definition in the following form

$$
E_{L L}=\frac{r}{2}(B-1)
$$

\section{Energy distribution in Weinberg's prescription}

The Weinberg's energy-momentum complex [7] is expressed as

$$
W^{\mu \nu}=\frac{1}{16 \pi} \Delta_{, \lambda}^{\mu \nu \lambda}
$$

where Weinberg's superpotential $\Delta^{\mu \nu \lambda}$ is antisymmetric on its first pair of indices which defines as

$$
\Delta^{\mu \nu \lambda}=\partial^{\mu} h_{\kappa}^{\kappa} \eta^{\nu \lambda}-\partial^{\nu} h_{\kappa}^{\kappa} \eta^{\mu \lambda}-\partial_{\kappa} h^{\kappa \mu} \eta^{\nu \lambda}+\partial_{\kappa} h^{\kappa \nu} \eta^{\mu \lambda}+\partial^{\nu} h^{\mu \lambda}-\partial^{\mu} h^{\nu \lambda}
$$


where $\partial_{\mu} \equiv \partial / \partial x^{\mu}, \partial^{\mu} \equiv \partial / \partial x_{\mu}$ and $h_{\mu \nu}$ shows the symmetric tensor defined as $h_{\mu \nu}=$ $g_{\mu \nu}-\eta_{\mu \nu}$, where $\eta_{\mu \nu}$ is the Minkowski metric. The energy in Weinberg's prescription for a four-dimensional background is given by

$$
E=\iiint W^{00} d x^{1} d x^{2} d x^{3}
$$

where $W^{00}$ is the energy density component. Using Gauss's theorem, one has

$$
E=\frac{1}{16 \pi} \iint \Delta^{i 00} n_{i} d S
$$

The energy distribution connected to the generic SSS metric in Weinberg's formulation becomes the same as the energy derived from Landau-Lifshitz's prescription, i.e.

$$
E_{W}=E_{L L}=\frac{r}{2}(B-1) .
$$

\section{Energy distribution in Papapetrou's prescription}

The energy and momentum in the prescription of Papapetrou [3] takes the form

$$
\Omega^{\mu \nu}=\frac{1}{16 \pi} N_{, \lambda \kappa}^{\mu \nu \lambda \kappa},
$$

with

$$
N^{\mu \nu \lambda \kappa}=\sqrt{-g}\left(g^{\mu \nu} \eta^{\lambda \kappa}-g^{\mu \lambda} \eta^{\nu \kappa}+g^{\lambda \kappa} \eta^{\mu \nu}-g^{\nu \kappa} \eta^{\mu \lambda}\right),
$$

where $N^{\mu \nu \lambda \kappa}$ is Papapetrou's superpotential and is symmetric on its first pair of indices. The energy in the Papapetrou prescription for a four-dimensional background is given by

$$
E=\iiint \Omega^{00} d x^{1} d x^{2} d x^{3}
$$

where $\Omega^{00}$ represents the energy density component. Using Gauss's theorem, the energy component is

$$
E=\frac{1}{16 \pi} \iint N_{, j}^{00 i j} n_{i} d S .
$$

Using the metric (66), we get the energy component of Papapetrou's definition in the following form

$$
E_{P}=\frac{r}{8(A B)^{\frac{3}{2}}}\left[4 A^{2} B(B-1)+r\left(A^{\prime} B^{2}-A B B^{\prime}-A A^{\prime} B+A^{2} B^{\prime}\right)\right],
$$

where the prime abbreviates $\partial / \partial r$. 


\section{E. Energy distribution in Møller's prescription}

The energy-momentum complex of Møller [8] is given by

$$
M_{\nu}^{\mu}=\frac{1}{8 \pi} \chi_{\nu, \lambda}^{\mu \lambda}
$$

where Møller's superpotential $\chi_{\nu}^{\mu \lambda}$ has the form

$$
\chi_{\nu}^{\mu \lambda}=-\chi_{\nu}^{\lambda \mu}=\sqrt{-g}\left(g_{\nu \sigma, \kappa}-g_{\nu \kappa, \sigma}\right) g^{\mu \kappa} g^{\lambda \sigma} .
$$

The energy component in Møller's prescription is given by

$$
E=\iiint M_{0}^{0} d x^{1} d x^{2} d x^{3},
$$

where $M_{0}^{0}$ is the energy density component. Using Gauss's theorem, the energy component is equal to

$$
E=\frac{1}{8 \pi} \iint \chi_{0}^{0 i} n_{i} d S .
$$

Note that the calculations are not anymore confined to quasi-Cartesian coordinates. Hence, we utilize the metric (5) to get the energy distribution in Møller's definition as follows:

$$
E_{M}=\frac{r^{2} A^{\prime}}{2 \sqrt{A B}} .
$$

It would be worthwhile to denote that, the energy given by Eqs. (12), (17), (22), (27) and (32) is also called the effective gravitational mass $M_{\text {eff }}$ of the spacetime under consideration. For instance, the energy distribution in Møller's prescription given by Eq. (32) is in fact the energy (effective mass) of the gravitational field that a test particle present at a finite distance $r$ in this field experiences. Studying on the problem of finding the effective gravitational mass was first considered by Cohen and Gautreau [18]. Afterwards, much attention has been devoted to this issue for different spacetimes [19].

In the following, we will present Verlinde's entropic scenario [16] to investigate the emergent gravitational energy on SSS screens.

\section{ENTROPIC GRAVITY}

In order to obtain the energy on a holographic screen for a generic SSS spacetime, we should find the timelike Killing vector of the metric (15). Using the Killing equation

$$
\partial_{\mu} \xi_{\nu}+\partial_{\nu} \xi_{\mu}-2 \Gamma_{\mu \nu}^{\lambda} \xi_{\lambda}=0
$$


with the condition of SSS, i.e. $\partial_{0} \xi_{\mu}=\partial_{3} \xi_{\mu}=0$, and also the infinity condition $\xi_{\mu} \xi^{\mu}=-1$, the timelike Killing vector is written as

$$
\xi_{\mu}=(-A, 0,0,0)
$$

To define a foliation of space, and distinguishing the holographic screens $\Omega$ at surfaces of constant redshift, we write the generalized Newtonian potential $\phi$ in the general relativistic framework

$$
\phi=\frac{1}{2} \log \left(-g^{\mu \nu} \xi_{\mu} \xi_{\nu}\right)=\frac{1}{2} \log A,
$$

where $e^{\phi}$ is the redshift factor and is equal to one at the reference point with $\phi=0$ at infinity. Thus, the acceleration $a^{\mu}$ for a particle that is placed close to the screen yields the following:

$$
a^{\mu}=-g^{\mu \nu} \nabla_{\nu} \phi=\left(0, \frac{A^{\prime}}{2 A B}, 0,0\right) .
$$

The temperature on the holographic screen is given by Unruh-Verlinde temperature that is connected to the proper acceleration of a particle near the screen and can be written as [16]

$$
T=-\frac{1}{2 \pi} e^{\phi} n^{\mu} a_{\mu}=\frac{e^{\phi}}{2 \pi} \sqrt{g^{\mu \nu} \nabla_{\mu} \phi \nabla_{\nu} \phi}
$$

where $n^{\mu}=\nabla^{\mu} \phi / \sqrt{g^{\mu \nu} \nabla_{\mu} \phi \nabla_{\nu} \phi}$ is a unit vector which is normal to the holographic screen and to $\xi_{\mu}$. The Unruh-Verlinde temperature for the metric (15) is simply achieved and reads

$$
T=\frac{1}{4 \pi} \frac{A^{\prime}}{\sqrt{A B}}
$$

On the SSS screen, $N$ bits of information are stored and the holographic information about the source material is encoded as $d N=d \mathcal{A}$, where $\mathcal{A}$ is the area of the screen. According to the equipartition law of energy, the energy $E$ is distributed on a closed screen of the constant redshift $\phi$. For further details and for example, we display the energy associated with a source mass $M$ located at the origin of the coordinate. According to the figure, the spherical holographic screen $\Omega$ with an equilibrium temperature $T$ and the total equipartition energy $E$ is placed at a distance of $R$ from the source mass. A test particle with mass $m$ is located near the screen $\Omega$. The energy is smoothly distributed over the occupied bits, and is equivalent to the source mass that would emerge in the part of space surrounded by the screen. The situation is depicted in Fig. 1,

The energy on the holographic screen $\Omega$ for a generic SSS spacetime becomes

$$
E=\frac{1}{4 \pi} \int_{\Omega} e^{\phi} \nabla \phi d \mathcal{A}=2 \pi r^{2} T
$$




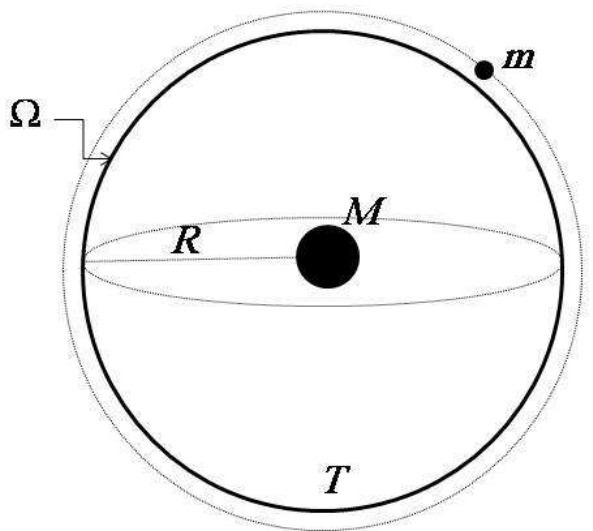

FIG. 1: A test particle with mass $m$ approaches the spherical holographic screen $\Omega$. The screen $\Omega$ possesses an equilibrium temperature $T$ and the total equipartition energy $E$ which is located at a distance of $R$ from the source mass $M$ at the origin. It is supposed that the energy associated with the source mass is evenly dispersed on the screen.

This result is in agreement with the Gauss's law. Using Eq. (38), the energy on the screen then takes the form

$$
E=\frac{r^{2} A^{\prime}}{2 \sqrt{A B}},
$$

which is exactly the same result as in the pseudotensor scenario that we have derived from the Møller definition of energy. This shows the importance of Møller's prescription for interpreting the energy distribution in GR [9].

\section{SUMMARY}

In summary, using Einstein, Landau-Lifshitz, Weinberg, Papapetrou and Møller energymomentum complexes, respectively, we have computed the energy distributions associated with the SSS gravitational background. In this way the effective gravitational mass experienced by a test particle situated at any finite radial distance in the gravitational field is found. On the other hand, using the emergent view of gravity we have obtained the emergent gravitational energy on a SSS screen. Our results show that the emergent gravitational energy obtained from the Verlinde approach is identical to the gravitational energy derived from the Møller approach. It seems that these two approaches possess similar behaviors. In both approaches, the energy is in fact contained in a two-sphere of radius $r$ which 
gives a taste of the effective gravitational mass that a test particle experiences. However, the other prescriptions yield the different results. This may lead to the approvement of Lessner's argument concerning the significance of Møller's prescription in the context of GR.

[1] A. Einstein, Preuss. Akad. Wiss. Berlin 47, 778 (1915); Addendum-ibid. 47, 799 (1915).

[2] R. C. Tolman, Relativity, Thermodynamics and Cosmology, Oxford University Press London p. $227(1934)$

[3] A. Papapetrou, Proc. R. Ir. Acad. A 52, 11 (1948).

[4] L. D. Landau and E. M. Lifshitz, The Classical Theory of Fields, Addison-Wesley Press Reading MA p. 317 (1951).

[5] P. G. Bergmann and R. Thompson, Phys. Rev. 89, 400 (1953).

[6] J. N. Goldberg, Phys. Rev. 111, 315 (1958).

[7] S. Weinberg, Gravitation and Cosmology: Principles and Applications of General Theory of Relativity, Wiley New York p. 165 (1972).

[8] C. Møller, Ann. Phys. (N.Y.) 4, 347 (1958).

[9] G. Lessner, Gen. Rel. Grav. 28, 527 (1996).

[10] E. C. Vagenas, Int. J. Mod. Phys. D 14, 573 (2005).

[11] S. S. Xulu, arXiv:hep-th/0308070].

[12] There is a large literature on this subject; see for instance, K. S. Virbhadra, Pramana 38, 31 (1992); N. Rosen and K. S. Virbhadra, Gen. Rel. Grav. 25, 429 (1993); K. S. Virbhadra, Pramana 44, 317 (1995); Pramana 45, 215 (1995); J. M. Aguirregabiria, A. Chamorro and K. S. Virbhadra, Gen. Rel. Grav. 28, 1393 (1996); S. S. Xulu, Int. J. Theor. Phys. 37, 1773 (1998); K. S. Virbhadra, Phys. Rev. D 60, 104041 (1999); Mod. Phys. Lett. A 15, 1511 (2000); M. Sharif, Int. J. Mod. Phys. A 17, 1175 (2002); E. C. Vagenas, Mod. Phys. Lett. A 21, 1947 (2006); T. Multamaki, A. Putaja, E. C. Vagenas and I. Vilja, Class. Quant. Grav. 25, 075017 (2008); S. S. Xulu, Int. J. Theor. Phys. 46, 2915 (2007); A. M. Abbassi, S. Mirshekari and A. H. Abbassi, Phys. Rev. D 78, 064053 (2008); M. Sharif and M. F. Shamir, Gen. Rel. Grav. 42, 1557 (2010); A. Tyagi, K. Sharma and P. Jain, Chin. Phys. Lett. 27, 079801 (2010); Z. Nourinezhad, and S. H. Mehdipour, Indian J. Phys. 86, 919 (2012). 
[13] T. Jacobson, Phys. Rev. Lett. 75, 1260 (1995).

[14] T. Padmanabhan, Mod. Phys. Lett. A 25, 1129 (2010).

[15] T. Padmanabhan, Rept. Prog. Phys. 73, 046901 (2010).

[16] E. P. Verlinde, JHEP 1104, 029 (2011).

[17] There is a large literature on this subject; see for instance, L. Smolin, arXiv:1001.3668] R. Banerjee, Int. J. Mod. Phys. D 19, 2365 (2010); P. Nicolini, Phys. Rev. D 82, 044030 (2010); R. G. Cai, L. M. Cao and N. Ohta, Phys. Rev. D 81, 061501 (2010); M. Li and Y. Wang, Phys. Lett. B 687, 243 (2010); C. Gao, Phys. Rev. D 81, 087306 (2010); Y. Ling and J. P. Wu, JCAP 1008, 017 (2010); Y. Tian and X. N. Wu, Phys. Rev. D 81, 104013 (2010); Y. S. Myung and Y. W. Kim, Phys. Rev. D 81, 105012 (2010); Y. X. Liu, Y. Q. Wang and S. W. Wei, Class. Quant. Grav. 27, 185002 (2010); E. Chang-Young, M. Eune, K. Kimm and D. Lee, Mod. Phys. Lett. A 25, 2825 (2010); Mod. Phys. Lett. A 26, 1975 (2011); K. Nozari and S. Akhshabi, Phys. Lett. B 700, 91 (2011); D. A. Easson, P. H. Frampton and G. F. Smoot, Phys. Lett. B 696, 273 (2011); S. Gao, Entropy 13, 936 (2011); A. Kobakhidze, Phys. Rev. D 83, 021502 (2011); B. L. Hu, Int. J. Mod. Phys. D 20, 697 (2011); M. Visser, JHEP 1110, 140 (2011); S. H. Mehdipour, Eur. Phys. J. Plus 127, 80 (2012); S. H. Mehdipour and A. Keshavarz, Europhys. Lett. 98, 10002 (2012); S. H. Mehdipour, Astrophys. Space Sci. 345, 339 (2013).

[18] J. M. Cohen and R. Gautreau, Phys. Rev. D 19, 2273 (1979); J. M. Cohen and F. de Felice, J. Math. Phys. 25, 992 (1984).

[19] See for instance, R. Kulkarni, V. Chellathurai and N. Dadhich, Class. Quant. Grav. 5, 1443 (1988); K. S. Virbhadra, Phys. Rev. D 41, 1086 (1990); Phys. Rev. D 42, 2919 (1990); K. S. Virbhadra and J. C. Parikh, Phys. Lett. B 317, 312 (1993); E. C. Vagenas, Int. J. Mod. Phys. A 18, 5781 (2003); Int. J. Mod. Phys. A 18, 5949 (2003); Mod. Phys. Lett. A 19, 213 (2004); Int. J. Mod. Phys. D 14, 573 (2005); S. H. Mehdipour, Can. J. Phys. 91, 242 (2013). 